\title{
Electroluminescence in polymer films
}

$\mathrm{O}$ ptically pumped lasers, using conjugated polymers as their active material, have been reported both in solution ${ }^{1-3}$ and in thin films $s^{4,5}$. There is now a great deal of interest in the prospects of producing similar, electrically pumped laser diodes ${ }^{1-6}$. Here we report tunnelling-induced electroluminescence experiments on polymer films which indicate that construction of such devices should be possible.

Microcavity devices ${ }^{5}$ have the appropriate structure for laser diode operation, with in-phase reflection from the cavity mirrors providing the necessary feedback. The role of superfluorescence remains a subject of debate ${ }^{6}$, but more important is the question of whether electrical injection can generate the required excitation densities for lasing to occur. From the threshold optical pump power for the establishment of gain, Hide et al. ${ }^{4}$ estimate that a current density in excess of $10^{6} \mathrm{~A} \mathrm{~m}^{-2}$ will be needed. This is much higher than is normally sustainable within an electroluminescent light-emitting diode (LED), and some have argued that it is fundamentally inaccessible ${ }^{7}$.

We note that electroluminescence can be generated by tunnelling injection from a scanning tunnelling microscope (STM) tip. In our own ultra-high vacuum experiments with thin films (approximately $3 \mathrm{~nm}$ thick) of poly(1,3-phenylene vinylene-co2,5-dioctyloxy-1,4-phenylene vinylene) on a gold surface, we observe electroluminescence at a tunnelling current of $100 \mathrm{pA}$ for an STM tip bias of $-2.5 \mathrm{~V}$ relative to the gold anode. Although the electric field at the sharp tip is relatively high, at this tip bias the mean field across the polymer film is approximately $10^{7} \mathrm{~V} \mathrm{~cm}^{-1}$, not significantly greater than used in many organic LEDs $^{8}$. Electrons from the tip can tunnel directly into unoccupied states of the polymer, and on combination with holes injected from the anode, excitons are formed that can decay radiatively with the emission of light.

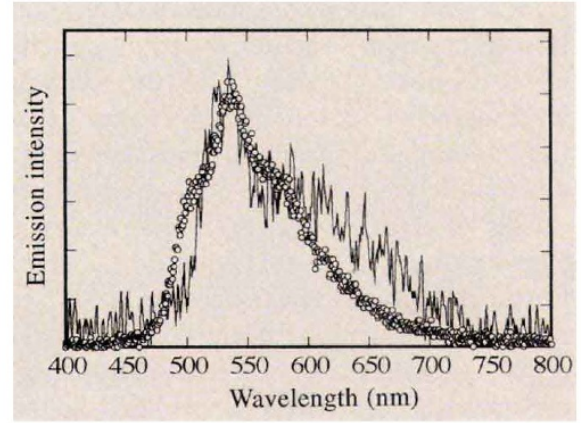

Figure 1 STM-induced electroluminescence emission spectrum (black line) and cathodoluminescence spectrum (circles), in arbitrary units.

The electroluminescent emission was collected and dispersed (Fig. 1), and was stable over periods of at least several minutes (the course of the experiment). The equivalence of the cathodoluminescence spectrum (recorded by raising the STM tip $\sim 1 \mu \mathrm{m}$ above the same spot on the surface and applying a $-200 \mathrm{~V}$ bias, sufficient to cause field emission from the tip), confirms that the electroluminescence spectrum does indeed arise from the polymer film.

The STM tip was scanned over the surface of the film, and the electroluminescence intensity and topography were simultaneously recorded as a function of position. The smallest features (less than $1 \mathrm{~nm}$ for a tunnelling current of $100 \mathrm{pA}$ ) resolved in the topographic image provide an upper limit of the tunnelling spot size, an assertion confirmed by the observation of intensity variations in the photon image over similar length scales. Because of the thinness of the film $(\sim 3 \mathrm{~nm})$ and the magnitude of the electric field, we expect little spreading within the film of the current injected through this spot. Hence we conservatively estimate that the applied STM tunnelling current of $100 \mathrm{pA}$ flows through an area of roughly $1 \mathrm{~nm}^{2}$, corresponding to a current density of $10^{8} \mathrm{~A} \mathrm{~m}^{-2}$. This exceeds by two orders of magnitude the estimate in ref. 4 . It indicates that there is no intrinsic limit to achieving electroluminescent emission at current densities which (in an optimized device structure) should satisfy the conditions for the establishment of optical gain.

The electroluminescent emission is highly non-uniform on length scales of $10 \mathrm{~nm}$ and greater, with variations in both emission intensity and spectral characteristics. Non-uniform emission occurring at similar current densities has also been observed in thin films of poly ( $p$-phenylene vinylene $)^{9}$, indicating that these results might apply to a large class of conjugated polymers. This non-uniformity in electroluminescence is problematic for device fabrication, and a detailed understanding of the processes that control local emission efficiency is required as part of any development of electrically pumped polymer lasers. Clearly there is still much to do in engineering a polymer laser diode, but we are convinced that this is an attainable target.

David G. Lidzey

Donal D. C. Bradley

Department of Physics,

University of Sheffield,

Hicks Building, Hounsfield Road,

Sheffield S3 7RH, UK

Santos F. Alvarado

Paul F. Seidler

IBM Research Laboratory, Säumerstrasse 4, $\mathrm{CH}-8803$ Rüschlikon,Switzerland

\footnotetext{
. Holzer, W., Penzkofer, A., Gong, S. H., Bradley, D. D. C. \& Bleyer, A. Adv. Mater: 8, 974-978 (1996).

Moses, D. Appl. Phys. Lett. 60, 3215-3216 (1992)

3. Brouwer, H. J., Krasnikov, V. V., Hilberer, A., Wildman, J.

\& Hadziioannou, C. Appl. Phys. Lett. 66, 3404-3406 (1995).

4. Hide, F. et al. Science 273, 1833-1836 (1996).

5. Tessler, N., Denton, G. J. \& Friend, R. H. Nature 382, 695-697 (1996).

6. Bradley, D. D. C. Nature 382, 671 (1996).

7. I.ovinger, A. J. \& Rothberg, L. J. J. Mater. Res. 11, 1581-1592 (1996).

8. O'Brien, D., Weaver, M. S., Lidzey, D. G. \& Bradley, D. D. C. Appl. Phys. Lett. 69, 881-883 (1996).

9. Alvarado, S. F, Reiss, W., Seidler, P. F. \& Strohriegl, P. Phys. Rev. $B$ (submitted).
}

\section{Adaptation of colour vision to sunlight}

The processes of light and dark adaptation in photopic vision are generally thought to be complete within minutes. Even after nearly all the photopigment in the retinal cone cells of the eye has been bleached by exposure to an intense light, both the density of pigment and the observer's sensitivity are said to recover fully in only 7 minutes $^{1}$. We describe here a much slower process, which reveals itself in an alteration of colour vision after an hour's adaptation to English sunlight.

Before and after exposure to natural sunlight, we measured Rayleigh matches, in which red and green spectral lights are mixed to match a monochromatic orange ${ }^{2}$. Using a Nagel anomaloscope, in which two semicircular fields are viewed simultaneously (see Fig. la), a subject adjusted the red/green ratio of the upper half-field to match the standard orange field. Data were also obtained with a computer-controlled colorimeter that allowed a $2^{\circ}$ red/green mixture field $(690+550 \mathrm{~nm})$ to be alternated every $2 \mathrm{~s}$ with a monochromatic orange field $(590 \mathrm{~nm})$, thresholds being determined with a randomized 'double-staircase' procedure (Fig. 1b). The exit pupils of both instruments were less than $1 \mathrm{~mm}$ in diameter and so variations in the natural pupil are unlikely to affect the stimulus.

During the adaptation phase of the experiment the subject read scientific papers illuminated by natural summer sunlight in central Cambridge (typically 80,000 lux). After exposure, Rayleigh matches were shifted in the protan direction - more red 